\title{
September 11, 2001, Timeline
}

\author{
A.M. \\ 8:46:40 American Airlines Flight 11 crashes into 1 World Trade Center \\ (the North Tower) \\ 08:47 MTA subway operator alerts MTA Subway Control Center of \\ an explosion in the WTC and begins emergency procedures \\ 08:52 PATH trains begin emergency procedures and proceed to evac- \\ uate WTC station and return Manhattan-bound trains to New \\ Jersey \\ 09:02:59 United Airlines Flight 175 crashes into 2 World Trade Center \\ (the South Tower) \\ 09:10 U.S. Coast Guard closes Port of New York and New Jersey \\ Port Authority of NY and NJ closes all its bridges and tunnels \\ 09:17 FAA shuts down all NYC airports \\ 09:37:45 American Airlines Flight 77 hits the west wall of the Pentagon \\ 09:58:59 South Tower collapses \\ 10:03:11 United Airlines Flight 93 crashes in Pennsylvania \\ 10:20 NYC Transit suspends all subway service \\ 10:28:22 North Tower collapses \\ 10:30 NJ Transit stops rail service into Manhattan's Penn Station \\ 10:45 U.S. Coast Guard calls for "all available boats" to assist evacua- \\ tion of Lower Manhattan; PATH operations suspended \\ 11:02 Mayor Giuliani calls for evacuation south of Canal Street
}

\section{P.M.}

05:20 7 World Trade Center, headquarters of NYC Office of Emergency Management, collapses

Sources:

U.S. Department of Transportation's John A. Volpe National Transportation Systems Center, "Effects of Catastrophic Events on Transportation System Management and Operations: New York City-September 11," 2/6/17, https://ntl.bts.gov/lib/jpodocs/repts_te/14129.htm.

U.S. Department of Commerce, Technology Administration, National Institute of Standards and Technology, "Final report on the collapse of the World Trade Center towers," 4/26/17, http://ws680.nist.gov/publication/get_pdf.cfm?pub_id=909017.

P.J. Capelotti, Rogue Wave: The U.S. Coast Guard on and after 9/11, Washington, DC, U.S. Coast Guard Historians Office, 4/27/17, https://www.uscg.mil/history/91101/pubs/ 911_Rogue_Wave.pdf. 
1 Port Imperial

2 Intrepid Sea, Air, and Space Museum (Pier 84)

3 Piers 81-83

4 Jacob K. Javits Convention Center

5 Lincoln Harbor

6 Pier 63

7 Pier 62

8 Pier 61

9 Pier 60

10 Pier 59

11 Pier 53

12 Long Slip

13 Pier 40

14 Downtown Boathouse (Pier 26)

15 PATH Tubes (Formerly H\&M Hudson Tubes)

16 Paulus Hook

17 Colgate Clock

18 Morris Canal

19 Liberty Landing Marina

20 Liberty State Park

21 Red Hook Container Terminal

22 South Street Seaport

23 Brooklyn Navy Yard

24 North Cove

25 Whitehall Terminal (Staten Island Ferry)

26 World Trade Center complex

271 World Trade Center (Tower One) (North Tower)
282 World Trade Center (Tower Two) (South Tower)

293 World Trade Center

304 World Trade Center

315 World Trade Center (Borders Books and Music)

326 World Trade Center

337 World Trade Center

341 World Financial Center

352 World Financial Center

363 World Financial Center

374 World Financial Center

38 Winter Garden

39 World Financial Center Terminal

40 North Pedestrian Bridge

41 South Pedestrian Bridge

42 Gateway Plaza (VIP Yacht Cruises offices and St. Joseph's Chapel on the first floor)

43 Albany Street townhouse

44 Zuccotti Park

45 New York Stock Exchange

46 South Cove

47 Robert F. Wagner Jr. Park

48 Pier A

49 The Battery

50 Battery Park

51 U.S. Coast Guard offices

52 Downtown Heliport

53 Pier 11 